\title{
"A la conquista de la opinión pública!": eugenesia latina a través de la revista Viva Cien Años, Argentina, 1934-1947
}

\author{
"Conquer public opinion!:" Latin American eugenics as seen \\ in the magazine Viva Cien Años, Argentina, 1934-1947
}

\section{Luciana Mercedes Linares}

Profesora, Departamento de Historia/Universidad Nacional de Mar del Plata.

Mar del Plata - Buenos Aires Argentina

lume_linares@hotmail.com
LINARES, Luciana Mercedes. "A la conquista de la opinión pública!": eugenesia latina a través de la revista Viva Cien Años, Argentina, 19341947. História, Ciências, Saúde - Manguinhos, Rio de Janeiro, v.25, supl., ago. 2018, p.179-192.

\section{Resumen}

La eugenesia como disciplina desembarcó en Argentina a principios del siglo XX. Contó con una amplia recepción entre intelectuales y profesionales de la salud, en medio de una serie de acciones tendientes a fortalecer redes tanto dentro del mundo académico como político. Entre las iniciativas surge, en 1934, la revista Viva Cien Años, como una publicación de carácter científico y popular, en intenciones. Este artículo presenta las posibilidades del corpus documental referido a la revista, publicada en Argentina hasta 1947. El eje central propone vincular la aparición de la publicación con la impronta de la eugenesia de los años 1930 en la Argentina y las estrategias tendientes a la conquista de la opinión pública.

Palabras clave: Viva Cien Años (revista); eugenesia; medios de comunicación; opinión pública.

\section{Abstract}

Eugenics arrived in Argentina as a discipline at the beginning of the twentieth century. It was widely embraced by intellectuals and healthcare professionals, in the midst of a series of actions undertaken to strengthen networks within the academic and political worlds. One of those initiatives was the magazine Viva Cien Años, founded in 1934 as a popular science publication. This article presents the possibilities of the body of documentation relating to the magazine, which was published in Argentina until 1947. The central thesis argues that the appearance of this publication shows the impact of eugenics in the 1930s in Argentina, and its strategies for conquering public opinion.

Keywords: Viva Cien Años (magazine); eugenics; communication media; public opinion. 


\section{La construcción de la otredad en la revista Viva Cien Años}

Aproximarnos al estudio de la eugenesia latina desde el análisis del corpus documental de la revista Viva Cien Años (VCA) abre la posibilidad de avanzar en el conocimiento de las prácticas de difusión de la propaganda eugénica y de sus intentos por lograr penetrar en la opinión pública. Para analizar estos canales - tanto en objetivos como en prácticas resultan relevantes los mecanismos de construcción del discurso hacia el público general a partir de secciones, editoriales, fotografías, cartas de lectores y publicidad en VCA durante el periodo 1934-1947.

La publicación en cuestión contaba con auspicios institucionales, además de una variada publicidad comercial. Entre estos avales institucionales, encontramos prestigiosas asociaciones profesionales y organismos gubernamentales de distintos países americanos. Podemos mencionar entre otros, la Asociación Médica Argentina, Asociación Paulista de Medicina, Instituto de Pesquisas Juveniles de São Paulo, Ministerio de Educación y Salud Pública de Brasil, Ministerio de Salubridad, Previsión y Asistencia Social de Chile, Departamento Nacional de Higiene de Colombia, Liga Argentina de la Higiene, Museo Social Argentino, Ministerio de Justicia y Acción Pública de Argentina. En sus números, estas instituciones se veían representadas por distintos colaboradores que describían la situación sanitaria de sus países, referían a innovaciones en el campo de la medicina, como a distintas necesidades de la vida cotidiana, dejando entrever, en todas esas manifestaciones, las ansiedades acerca de las posibilidades de mejoramiento de la raza en el continente americano.

Dentro de estas operaciones, nos resulta interesante pensar en la construcción discursiva de la otredad que rige en la revista. En dicha construcción existió una complicidad de discursos. Es decir, una confluencia y circulación de ideas sobre el tema que se encuentra encendido en la agenda de figuras de diferentes campos, como el jurídico, el médico, el político, etc. Tales construcciones permiten observar de qué manera se interviene en la cuestión social para crear e imponer legitimidad en los discursos (Angenot, 2010, p.67). La sociedad de los años 1930 atravesaba un cambio de fisonomía en cuanto a su estructura poblacional y a la vertiginosa vida política. Así también, esta década fue testigo de la consolidación del proceso de medicalización y consecuentemente de la legitimación social de una corporación, con incidencia en la vida privada de las personas: la médica.

Los marcos interpretativos y nuevos paradigmas referidos a la medicina social, evidenciaron el clima de época y la indudable aparición de variables de orden político que se plasmaron en dichas teorías. Así se fue forjando una teoría social que vinculó la política y la biología en un mismo campo que tendría como fin la selección del "nuevo hombre". La determinación de lo que compone un "nosotros" y "aquello", dado por la otredad, lo dirime un proceso que involucra cuatro componentes: la identificación, la clasificación, la jerarquización y la exclusión del individuo (Miranda, 2011). La revista VCA introduce estas nociones que, a su vez, construyeron el sentido de legitimidad necesario para catalogar ese "otro" que pone en peligro la posibilidad sudamericana de mejoramiento racial. 


\section{Viva Cien Años, en el contexto socio-histórico}

La publicación VCA surgió en Argentina hacia mediados de la década de 1930 como un emprendimiento editorial dirigido por un grupo de médicos con una reconocida trayectoria en ámbitos académicos y científicos. Con el auspicio de varias instituciones reconocidas argentinas y latinoamericanasy diversos profesionales de la salud, VCA se lanzó al mercado editorial con la impronta de ser la primera revista de divulgación popular de la salud en Sudamérica. En principio, mensual y luego quincenal, la revista contaba también con el apoyo de publicidad comercial variada que luego sería monopolizada por distintos laboratorios. Fue la primera publicación que daría lugar a un sello editorial que tuvo no solo otra revista, sino programas radiales, libros, folletines, ciclo de charlas y conferencias y hasta una escuela de salud. Así Arturo León López, Mariano Barilari y Godofredo Grasso, comprometidos con la eugenesia en su versión criolla y con la higiene mental dieron el puntapié inicial del surgimiento de medios de comunicación especializados en medicina pero de divulgación popular. El consejo médico y su carácter de verdad por el bien común fue el principal mecanismo de penetración en el hogar de los lectores-pacientes en pleno boom del comercio editorial popular (Rocchi, 1999). Los fundadores de la revista provenían de diferentes campos y con recorridos diversos pero con la misma preocupación de "generar un órgano público que llevará al pueblo palabra cierta y orientadora de los hombres de ciencia ... teniendo como norte mejorar al individuo, consolidar a la familia y fortificar la raza, cuyo vigor es la base de toda grandeza nacional" (López, 1 oct. 1944, p.88). López fue kinesiólogo, ávido autor de revistas especializadas, buscó el apoyo de una eminencia de la Facultad de Medicina de la Universidad de Buenos Aires (UBA), como lo fue Julio Iribarne, y consiguió vincularse con el Museo Social Argentino. Fue Iribarne quien oficiaría de nexo entre él, Barilari y Grasso. Mariano Barilari inició su formación en medicina en la UBA y la culminó en Alemania durante la Primera Guerra Mundial. Entendió a la medicina como un campo multidisciplinario y consideraba importante repensar la faz orgánica y psíquica de las personas desde un criterio funcional. Grasso fue médico kinesiólogo, con diversos reconocimientos de instituciones internacionales en su campo y también un profesional reconocido por sus aportes y artículos en revistas y boletines especializados. Entre los profesionales de la salud que escribían en la revista sus artículos con las últimas investigaciones para la década de los años 1940 sobre cáncer y temas como obesidad, psicología, malaria, entre otras temáticas para América Latina, podemos mencionar a Federico Bellisio, Antonio Pita, Carlos Padilla Roqué, Lucian Daniells, Jaime Bertrán, Thalino Botelho, Honorio Delgado.

Desde su título, la revista VCA planteaba una propuesta clara: dar respuesta a las cuestiones poblacionales desde el cuidado de la herencia de genes y desarrollo de pautas eugénicas en la vida cotidiana de las personas. Así, planteaba en notas editoriales, columnas, artículos y publicidad comercial, la posibilidad de que América Latina "optimizara" su "capital humano", no desde una obra estatal de amplias dimensiones, sino desde las conductas de las personas. El sujeto dinamizador de la eugenesia como ciencia práctica, sería el individuo, en él estaba concentrada la posibilidad de cambio, siguiendo una suerte 
de recetario eugénico. La cuestión clave en esta publicación estuvo dada por los mecanismos utilizados a la hora de diseñar las estrategias comunicacionales.

Presente en las principales capitales provinciales del país, VCA alentaba a sus lectores, en particular a las mujeres, a constituirse en sujetos activos en esta cruzada biopolítica en pos de la mejora racial. No solo como agentes difusores de la eugenesia práctica en sus hogares, sino como concientizadores y replicadores. Desde temáticas múltiples, como el matrimonio (Vezzetti, 1985), el deporte, el psicoanálisis y psiquiatría (Weissmann, 2002), la alimentación (Schencman, 2010), el rol de la mujer (Linares, 2014) la revista, que se proponía ser una "publicación científico higiénica, esencialmente popular" - slogan que acompañaba el título -, planteaba la resolución, a partir del ideario eugenésico, de diferentes problemáticas como higiene, salud pública, enfermedades, saneamiento.

\section{Algunas cuestiones sobre los documentos}

Los documentos que exponemos aquí dan cuenta de los mecanismos mediante los cuales se presentan al lector-paciente las problemáticas sociales, en términos de población, y cómo se plantean las soluciones desde el marco de la eugenesia latina. La selección de estos cuatro documentos - entre más de doscientos números de la revista a lo largo de toda su trayectoria - pretende dar cuenta de instancias diferentes en las operaciones discursivas. Van desde un artículo, una nota de carácter celebratorio, una editorial reflexiva y finalmente una publicidad de difusión de actividades.

El primer documento (Escardó, nov. 1936), da cuenta de las estrategias de presentación de la eugenesia en contexto socio-histórico, como nueva respuesta a viejos problemas de la sociedad, así también como la coexistencia de discursos profesionales en función del pro-natalismo. Florencio Escardó, su autor, fue un pediatra reconocido y comprometido con los ideales sanitaristas. Fue decano de la Facultad de Medicina de la UBA y vicerrector de esa universidad, años más tarde.

Más adelante, podemos evidenciar la puesta en marcha de redes entre los eugenistas argentinos y los principales referentes en América. Un caso paradigmático de ello es el de Renato Kehl, quien tuvo un rol protagónico en el armado de redes internacionales durante el período de entre guerras (Souza, 2007). Además de haber sido director de la filial brasilera de Industria Bayer - uno de los auspiciantes comerciales de la revista VCA hasta entrada la década del 1940 - escribía artículos que vinculaban la cuestión social con la responsabilidad eugénica. Su artículo (Kehl, ene. 1938) - de carácter celebratorio - evidencia también cómo profesionales que están vinculados a organizaciones internacionales, pusieron el acento en los mecanismos comunicacionales y el rol de los medios de comunicación para el propósito de la eugenesia latina.

El tercer documento (Editorial..., ene. 1939) es una editorial de la revista que abre el primer número del año 1939, donde se refuerza esta idea de las posibilidades de América de dar respuesta a través de un modelo eugenista e introduce, en conceptos simples, la idea de "nuevo hombre" como núcleo de la "raza fuerte" y aquel "otro" que pone en peligro ese ideal. 
El último documento (Publicidad, 1 oct. 1941) refiere a una publicidad que da difusión a las actividades de la "escuela de salud" - perteneciente al proyecto orientación integral humana, que contenía las iniciativas comunicacionales de los creadores de VCA que se presentaba como un espacio de capacitación para el público en general. Aquí se hace referencia directa a la eugenesia como disciplina práctica. Constituye otra de las estrategias para difundir la eugenesia como respuesta y ganar legitimidad en la opinión pública.

\section{REFERENCIAS}

ANGENOT, Marc.

El discurso social: los límites históricos de lo pensable y lo decible. Buenos Aires: Siglo XXI. 2010.

EDITORIAL...

Editorial: América debe adquirir una conciencia de su responsabilidad. Viva Cien Años, v.6, n.8, p.511. ene. 1939.

ESCARDÓ, Florencio.

¡Eugenesia! "Los padres comieron uvas ácidas y son los hijos los que tienen los dientes corroídos!". Viva Cien Años, v.3, n.2, p.133, 146147, 151. nov. 1936.

KEHL, Renato.

En el siglo de la eugenesia. Viva Cien Años, v.4, n.7, p.448-449, 497-498. ene. 1938.

LINARES, Luciana.

Delineando los cuerpos: construcciones discursivas en torno a la mujer a través de la propaganda eugénica en Argentina: el caso de la publicación 'Viva Cien Años', 1934-1947. Tesis (Maestría en Historia) - Universidad Nacional de Mar del Plata, Mar del Plata. 2014.

LÓPEZ, Arturo León.

Un recuerdo en la primera hora. Viva Cien Años, año 11, v.18, n.1, p.88. 1 oct. 1944.

MIRANDA, Marisa.

Controlar lo incontrolable: una historia de la sexualidad en la Argentina. Buenos Aires: Biblos. 2011.

PUBLICIDAD.

Publicidad. Viva Cien Años, v.12, n.1, p.56. 1 oct. 1941.
ROCCHI, Fernando.

Inventando la soberanía del consumidor: publicidad, privacidad y revolución del mercado en Argentina, 1860-1940. In: Devoto, Fernando; Madero, Marta. Historia de la vida privada en la Argentina, v.3. Buenos Aires: Taurus. p.301-321. 1999.

SCHENCMAN, Pablo.

¿Génesis de un problema sanitario?

Representaciones de la biomedicina en torno a la obesidad en Viva Cien Años, 1934-1945. Eä Journal, v.2, n.2, p.1-30. 2010.

SOUZA, Vanderlei Sebastião de.

La eugenesia de Renato Kehl y la formación de una red internacional en el período entreguerras. In: Vallejo, Gustavo; Miranda, Marisa (Comp.). Políticas del cuerpo: estrategias modernas de normalización del individuo y la sociedad. Buenos Aires: Siglo XXI. p.425-454. 2007.

VEZZETTI, Hugo.

"Viva Cien Años": algunas consideraciones sobre la familia y matrimonio en la Argentina. Punto de Vista, año 9, n.27, p.5-10. 1985.

WEISSMANN, Patricia.

Mariano J. Barilari: medicina psicosomática y educación para la salud en la Argentina de los años 30' (Primera parte). In: Red Iberoamericana de Historia de la Psiquiatría. Medicina psicosomática y educación para la salud en la Argentina de los años '30. (Temas de Historia de la Psiquiatría, n.15). Disponible en: http://www. investigacion.cchs.csic.es/rihp/Temas15. Acceso en: 16 ago. 2011. Primavera 2002.

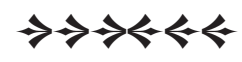




\title{
¡Eugenesia! "Los padres comieron uvas ácidas y son los hijos los que tienen los dientes corroídos!"
}

\author{
Por el dr. Florencio Escardó
}

La palabra hereditario lleva al ánimo de la mayoría de las gentes la noción de algo contra lo que no es posible luchar y ante lo cual toda previsión y toda defensa resultan inútiles. La herencia aparece como una diosa temible e inexorable que actúa con rigor ciego. En el curso de estos artículos nos hemos esforzado en demostrar cómo, a medida que han progresado los estudios médicos, muchas enfermedades que se consideraban hereditarias no lo son, de modo que la posibilidad de luchar contra ellas se hace posible y hacedera.

Esta manera de pensar se ha ido adentrando poco a poco en la educación general y una legión de hombres cultos y generosos está empeñada, en todo el mundo, en propagar ideas claras y firmes sobre la defensa de la especie humana, de modo que las nuevas generaciones comprendan que la salud es un don que puede merecerse y ganarse mediante una conducta inteligente e informada.

Este alto y noble interés por la especie no se detiene en el hombre presente, sino que aspira a salvaguardar y proteger a las generaciones futuras, buscando en ello hacer más justo y más bueno el porvenir de los hombres. Lo primero que ha procurado esta ideología ha sido cambiar la actitud de las gentes frente al problema de la herencia, incitando a cada uno a sentir la verdadera responsabilidad que nos incumbe con responsabilidad a ella.

En nuestro primer artículo dijimos que el problema de la herencia tenía dos polos: uno puramente científico, la genética y otro aplicado, la eugenesia.

De la eugenesia vamos ocuparnos hoy. Sin espacio suficiente para desarrollar el tema con toda la amplitud que merece, fijaremos aquí los jalones principales que la ciencia eugenésica ha colocado en su lucha por mejorar las condiciones humanas.

Lo primero que es preciso adoptar en este tren de ideas es lo que podríamos llamar "la actitud eugenésica". Esta actitud consiste en compenetrarse de que nadie tiene derecho a generar seres tarados en sus capacidades físicas o disminuidos en sus valores humanos. Y así como la conservación de la salud se nos aparece como una obligación natural hacia nosotros mismos, el deber generar hijos se nos debe aparecer como una obligación de derecho natural hacia la especie. Nadie puede discutir la lógica, la nobleza y justicia de este postulado.

Veamos ahora qué normas prácticas ha fundado la eugenesia para lograr tal resultado. El primer paso está realizado por lo que se llama el matrimonio eugenésico. Para lograrlo, los futuros padres deben en el momento en que dispongan a serlo someterse al examen prenupcial.

¿Qué es el examen prenupcial? Simplemente la práctica de realizar un balance de salud antes de contraer matrimonio. Así dicho, el asunto parece fácil por demás, pero en la práctica se muestra lleno de dificultades. Unas veces es falso pudor de que se sepa que padecen ciertas enfermedades, quienes tal vergüenza tienen, arriesgan que esa vergüenza le sea enrostrada por la vida en la cara o en la deformidad del hijo. Otros y no pocos, 
tienen el temor de ver certificada una dolencia y prefieren ignorarla y no oírla de boca del médico, la tendrán que soportar de boca de todo el mundo cuando la descendencia marcada la ponga en evidencia.

Otros creen que el médico niega o da permiso para contraer matrimonio y temen que una actitud negativa de su parte esté en contradicción con compromisos sociales o familiares. No hay nada de eso. Lo único que el médico da es la conciencia sobre el estado de la salud y por lo tanto indica las normas que deberán seguirse para que en caso de existir una enfermedad ésta no recaiga sobre los hijos. Ya hemos visto cómo los flagelos más temidos, la sífilis y la tuberculosis, son en gran medida susceptibles de ser ahorrados a los hijos de quienes las padecen.

Hay también personas que suponen que el examen prenupcial de las mujeres implicaría revisaciones intimas en pugna con el natural pudor. Nada más erróneo, el examen médico prenupcial no difiere de ninguna otra revisación médica corriente. En los países cuya legislación está lo suficientemente avanzada este examen es obligatorio por ley: esas leyes interpretan con verdad que la salud de las nuevas generaciones es una obligación de la que el Estado no puede desentenderse.

Entre nosotros, el examen prenupcial no ha pasado de una noble aspiración de los médicos preocupados de la medicina social; pero creemos que no está ya lejano el día en que su práctica entre en la mentalidad de las gentes como una obligación de conciencia.

Es curioso y en cierta manera vergonzoso que las familias se preocupen y en algunos países hasta estipulen contractualmente la situación económica de los fututos cónyuges, y en cambio no se haga ninguna tentativa seria por el conocer y garantizar el patrimonio biológico de dos seres cuya salud va a repercutir sobre terceros que no tienen más defensa que la conciencia de quienes le van a dar el ser.

Este examen prenupcial representa el desiderátum de la defensa, puesto que es como una avanzada colocada en el primer puesto de peligro, pero cuando su misión no se cumple, la eugenesia postula otras formas que, con gran eficacia, pueden proteger la vida del ser futuro. Estos postulados consisten en el cuidado del niño desde el "primer momento en el seno de la madre"; una ciencia especial llamada puericultura prenatal, se ha dedicado al estudio particular de la salud del niño antes de nacer; en ese plazo en que la nueva vida transcurre en las entrañas maternas pueden tomarse muchas garantías para la salud futura; es en ese tiempo que las enfermedades transmisibles como la sífilis podrán ser combatidas, y los accidentes del nacimiento, que a menudo cuestan la vida al niño o lo taran seriamente, pueden ser planteados con el tiempo y resueltos con oportunidad.

Para ello es preciso que la madre se ponga bajo vigilancia especial desde el primer momento que se sienta embarazada y no solo acepte sino también solicite un reconocimiento completo y profundo de su salud; no por ella sino también por el grave deber que contrae con la vida nueva que lleva en sí.

Entre nosotros, la observación muestra que muchas madres esperan el último momento de su gravidez para procurarse asistencia; es preciso que sepan que en ese instante casi todo es irreparable y que el mejor arte y la más honda ciencia no podrán resolver situaciones que solo hubieran evitado con tiempo prudente; la misma observación señala que muchas madres se ponen bajo el consejo y cuidado de gente inexperta. Cuando hemos dicho repetidamente 
en estas páginas respecto a que la salud es un problema demasiado trascendental y grave para confiarse a aficionados, encuentra lugar de ser recordado aquí.

Muchas enfermedades de los niños y no pocas de las madres se pueden precaver con el cumplimiento de las prácticas de puericultura prenatal.

El buen sentido indica cuántas ventajas se obtienen al hacer control periódico de la salud. Como el ideal es que el médico sea el guardián de la salud y no el de la enfermedad, si este ideal no se cumple debemos al menos aprovechar las circunstancias en que nuestra capacidad biológica va a repercutir sobre terceros para tomar sobre ella todos los reparos y recaudos necesarios. Y el casamiento y la paternidad son dos trances de tal modos vinculados a la especie que parecen indicarse por sí solos como etapas obligatorias e inexcusables del balance de salud que señalamos aquí como uno de los postulados más sabios y fecundos de la eugenesia.

Esta disposición de ánimo y un continuo espíritu de educación higiénica harán que pueda quitarse el carácter tremendo de aquella frase de la Escritura que pesa como una maldición: "Los padres comieron uvas ácidas y son los hijos los que tienen los dientes corroídos" (Escardó, nov. 1936, p.133, 146, 147, 151; énfasis del original). 


\section{En el siglo de la eugenesia}

Por dr. Renato Kehl

Se ha descubierto que los degenerados e inferiores tienen más hijos, en tanto que los intelectuales son atacados de esterilidad. Es por eso que debe fomentarse la prolifidad entre los matrimonios de individuos superiores.

¿Cómo es posible evitar el desorden social cuando gran parte de la colectividad está compuesta de enfermos, de incapaces, de neurópatas, de semidementes y dementes, de amorales y de criminales?

Dice Carrel: "En las prisiones solo encontramos criminales poco inteligentes o mal equilibrados; los otros muchos más numerosos, viven en libertad, mézclanse con el resto de la población y proceden en tal forma que no solamente se libran de las prisiones, sino que, además, ridiculizan a los encargados de frenarlos".

La situación, especialmente en las grandes colectividades, se torna tan grave, que se admite, con toda franqueza, "que es imposible luchar con éxito contra el ambiente social viciado".

\section{Las causas del caos}

Las causas de este caos están al alcance de cualquier inteligencia lúcida, es innecesario que una "comisión" especial de "sabios" venga a decirnos en qué consiste y con qué remedios se cuenta para combatirlo. Nadie podrá negar que la vida artificial y artificiosa que vivimos transforma a muchas personas en enfermos mentales. Según Beers, en los EEUU, el número de individuos asilados actualmente a consecuencia de debilidad mental o locura es ocho veces mayor que el de enfermos tratados por tuberculosis en los hospitales; que cada año centenas de millares de nuevos locos y criminales deben ser recluidos en los establecimientos especializados; que millones de jóvenes de baja mentalidad están incapacitados para continuar sus estudios, y son candidatos a futuros desórdenes mentales; que es mucho mayor la fragilidad psíquica que la física, agravada por las complicaciones e irritaciones de la vida actual. ¿Cómo explica que haya más locos y débiles mentales entre los criminales que en el resto de la población; y del mismo modo, que la mayor parte de éstos se encuentre en libertad, siendo escasa la proporción de los psicópatas y criminales asilados, como señala Carrel, con justa y bien documentada razón, ¿por qué solo los tontos y los incapaces se dejan llevar a los tribunales? Trabajos cuidados y minuciosos sobre la constitución individual, el temperamento, las tendencias, las inclinaciones hereditariamente observadas en familias de individuos superiores, y también en familias de degenerados y criminales - demuestran que evidentemente la herencia predomina inexorablemente sobre todas las demás influencias. Los estudios realizados a gemelos univitelinos son patentes e indiscutibles a este respecto.

\section{Una conciencia eugénica}

He aquí pues, porque el combate de los desórdenes colectivos debe iniciarse con medidas que visen la defensa de la salud física, psíquica o mental de los individuos. Así - mediante 
la formación de la conciencia eugénica popular, con el auxilio de la escuela, la prensa y la radio - los cultores de la eugenesia y la higiene mental pretenden lograr su objeto. Mediante la difusión cultural en sus múltiples aspectos; mediante leyes eugénicas, en el sentido de restringir la proliferación de los infrahombres, de los semialienados y de los dementes; mediante la higiene del cuerpo y también del espíritu: "solo así se podrá esperar la cura paulatina del malestar universal".

Desde el punto de vista de la consolidación genotípica para bien de las generaciones futuras, todo lo demás no constituye sino simples paliativos inoperantes contra las fuentes mórbidas degenerativas, contra el deterioro constitucional y prenatal y contra todas las subsecuentes degeneraciones de orden psíquico y social.

Para atajar el mal: atacarlo, pues, de raíz

Es lo que proclaman los adeptos a la política eugenética. Programa eugénico y sus resultados: Todos los recursos educativos, espirituales, religiosos, no han conseguido hasta el presente, aplacar los instintos perversos y modificar el alma social.

Después de veinte siglos de doctrina metafísicas para el mejoramiento del hombre, todavía se repiten las guerras, las luchas por la conquista, los asesinatos, las atrocidades, las desarmonías sangrientas entre los individuos y los pueblos.

La verdadera causa de la degeneración individual y colectiva no ha sido expuesta aún con claridad y franqueza. Las causas de la decadencia del hombre individuo, lo mismo que la de los pueblos, reside, siempre, en último término, como dice Siemens, "en la falta de selección natural, que rechaza todas las otras causas a planos secundarios".

Al modificar esta barrera de selección del género humano, transtornóse el equilibrio físico y psíquico. La balanza se inclina cada día más hacia el platillo donde se acumulan los mediocres, los infelices, los degenerados, los criminales.

Contra todos esos elementos de nada valen las sanciones, según lo demuestran las ciencias y la experiencia; de casi nada vale la educación, porque ellos no están en condiciones de experimentar su influencia benéfica.

Los crímenes han aumentado en proporciones alarmantes, según lo demuestran las estadísticas de todas partes.

La locura toma un carácter inquietante. Los hospicios no pueden detener la marea ascendente. La gran masa desbordada inunda las ciudades, pueblos, aldeas y campos.

Los vicios a despecho de las recomendaciones religiosas, de las observaciones domésticas, de las enseñanzas escolares y los castigos legales, siguen multiplicándose.

La mayor fecundidad de los individuos inferiores e incapaces contrasta dolorosamente con la creciente limitación de la natalidad de los individuos fuertes, inteligentes y productivos.

La filantropía, el sentimentalismo, contrariando los preceptos selectivos naturales, salvando y favoreciendo la reproducción de los infra hombres, agravan aún más la decadencia y bastardeo del género humano.

En vista de lo expuesto, ¿qué hacer? ¿desistir de la lucha? ¿renunciar a la vida terrena? ¿esperar la otra vida? ¿recurrir a los Dioses? 
¡No!

Para modificar la situación social; para orientarla en beneficio de las generaciones futuras; para conseguir que la humanidad se componga de un superávit de elementos de paz y de trabajo, poner en práctica las medidas propuestas por la eugenesia.

¿Por qué?

Porque la selección eugénica de algunas generaciones será suficiente para ejercer por medio de la supresión de los malos troncos hereditarios una influencia considerable sobre el futuro de los pueblos.

El éxito de la eugenesia está ligado, ante todo, a la conquista de la opinión pública y en particular de las personas cultas.

Preposiciones eugenésicas: Todos los valores genotípicos, tanto de los individuospersonalidades como de las "colectividades", no solo se desprenden, sino que también se reflejan en las respectivas disposiciones hereditarias. En otros términos: el hombre es lo que es, el pueblo es lo que es, en virtud de lo que recibiera en herencia de sus antepasados.

La mayor amenaza para un individuo y para un pueblo consiste en la degeneración por el empobrecimiento de los caudales hereditarios. El individuo, lo mismo que el pueblo, "vale" de acuerdo con el valor de los caracteres recibidos hereditariamente.

Un pueblo se rebaja y degenera cuando dentro de un seno los tipos inferiores tienen más hijos que los capaces y bien dotados.

El mayor anhelo de los eugenistas consiste, pues, en la conservación de los linajes sanos; es decir en el aumento progresivo de las estirpes bien constituidas, de las familias compuestas por individuos inteligentes, equilibrados y activos.

Para alcanzar el fin arriba aludido el Estado deberá proporcionar a estas familias toda clase de facilidades y garantías. La eugenesia positiva quiere cualidad antes que cantidad. Por esto, sin abandonar a los incapaces, ha de cuidar con el mayor celo de los elementos valiosos y eugénicos de la nacionalidad.

El hombre moderno es contrario a la selección, según se realizaba entre los pueblos primitivos y aún se realiza entre tribus salvajes.

Después de una despreocupación racial casi absoluta, a fin de cuidar tan solo de la salvación del alma, el hombre moderno comienza a preocuparse del mejoramiento del cuerpo y la mente de la especie, por la selección racional.

Dice el profesor Simmens: De todos los conocimientos cuyo conjunto constituye las ciencias naturales, ninguno tiene, para la vida de la familia, de la raza y del Estado, una significación más profundas que las teorías de la herencia y de la selección y su aplicación práctica: la eugenesia o higiene de la raza. La introducción de la enseñanza de la biología general y de la eugenesia en los liceos, academias, colegios y escuelas; la creación de cátedras especiales de estas materias en las universidades; la anexión de estas teorías dentro de las enseñanzas (que son objeto de examen de derecho y economía política) constituye la reivindicación más permanente de la eugenesia.

Mil, dos mil años de civilización. En una progresión lenta y persistente a través de los campos cubiertos de cruces y de miserias, no han bastado para lavar el pecado original de Adán y Eva. 
Apóstoles, filósofos, hombres de ciencia han dado el máximo de su trabajo y de su resignación para descubrir el fiat salvator. Pero la humanidad, a pesar de los beneficios conquistados, continúa su marcha por la senda tortuosa de la degeneración.

Después de la propaganda y de la formación de la conciencia eugénica iniciase, al fin, la política eugénica.

Entramos, pues, en el siglo de la eugenesia (Kehl, ene. 1938, p.448-449, 497-498). 


\section{Editorial: América debe adquirir una conciencia de su responsabilidad}

No se ha desvanecido aún el eco de las conversaciones sostenidas en Lima entre los representantes de los países americanos. Consciente de su responsabilidad política y social, y de su segura gravitación de mañana en los asuntos del mundo entero, América despierta y comienza a prepararse para ese futuro que sabe próximo. ¡Magnífico! Eso demuestra que adquiere ya lo que podríamos llamar conciencia de su responsabilidad.

Empero, muchos nos tememos que se trata de una responsabilidad a medias: porque en efecto, no se trata meramente de un papel político y social el que está llamada a desempeñar. No debe olvidarse el elemento humano. Porque si América se olvida de él; si descuida la integridad física de sus habitantes, si desdeña considerar principalmente, y en primer lugar, sus deberes sanitarios, supoblación en una palabra, carece de la validez necesaria, ¿quién va a tomar sobre sus espaldas la tarea de afrontar aquella responsabilidad? ¿cómo puede un cuerpo enclenque afrontar una labor de ciclopes? En estos momentos, la mirada del Universo se encuentra posada sobre América. El Viejo Mundo se debate entre violentas convulsiones, en tanto que el Nuevo Mundo surge sereno y poderoso. ¡Pero cuidado! Algunas voces, y no alarmistas, se han levantado para señalar hechos que no coinciden por cierto con las características que pertenecen a los pueblos nuevos. Particularizándonos con la Argentina, hemos de recalcar especialmente la notable disminución del crecimiento vegetativo de la población. ¿Qué no es un fenómeno aislado, sino universal? Sin duda. Pero no es posible aceptarlo con resignación en América, pueblo joven cuya sangre debe ser por lo menos, tan generosa y rica como su suelo y su fecundidad no menor que la proverbial de sus pampas. Si América está dispuesta a aceptar el papel que le corresponde como paladín de la futura civilización, es importante que comience por examinar la validez de sus miembros. El concepto de salud es prácticamente inseparable del de las grandes obras. Y si la que corresponde a América en el futuro lo es, sus habitantes deben comenzar a cuidar su salud física para que sus hijos sean sanos y gallardos exponentes de una raza en cuyo seno pueda incubarse el superhombre que quería Nietzsche (Editorial..., ene. 1939, p.511). 


\section{[Publicidad]}

\section{Reabre sus puertas la "Escuela de la Salud"}

Conferencias para este mes. Para los padres y todos los que esperen serlo dignamente una vez. A partir del corriente mes se realizará en nuestro local de la calle Rivadavia 3246 y bajo los auspicios de la Escuela de la Salud, un ciclo de disertaciones a cargo de distinguidos especialistas, colaboradores de nuestras páginas, sobre temas cuya importancia surge del simple enunciado: Alimentación, por una parte; conocimiento de las leyes que deben regir una concepción normal, por otra.

Como de costumbre en todas ellas campeará un estilo llano, desprovisto por completo de tecnicismos de difícil comprensión. Además se referirán en todo momento a cuestiones prácticas de tal manera que usted pueda obtener de ellas la mayor utilidad posible.

\section{Eugenesia Práctica}

El mínimo de conocimientos que debe poseer un adulto sobre la generación normal. El problema de los hijos antes de la concepción, durante la gestación y después del nacimiento. Desde el punto de vista higiénico, moral y económico. A cargo del dr. Carlos M. Padilla Roque. Días Martes 7, 14, 21 y 28.

\section{Fundamentos de la Nutrición Humana}

Las leyes de la alimentación. Cuáles son los materiales con los que se construye el hombre y en qué cantidades deben figurar en el diario "menú". La alimentación normal del niño y del adulto de ambos sexos. A cargo del Médico Federico B. Bellisio. Días Viernes 10, 17, 24 y 31 (Publicidad, 1 oct. 1941, p.56). 